\title{
TYPE II SUPERNOVAE IN BINARY SYSTEMS
}

\author{
P.C. JOSS \\ Massachusetts Institute of Technology \\ Room 6-203, Cambridge MA 02139, U.S.A.
}

\begin{abstract}
The presence of a close binary companion can affect the evolution of a massive star through one or more episodes of mass transfer, or by merger in a common-envelope phase. Monte Carlo calculations indicate that $\sim 20-35 \%$ of all massive supernovae are affected by such processes, and that a substantial fraction of these events will be supernovae of type II. The properties of the progenitor star, the distribution of circumstellar material, the peak supernova luminosity, the shape of the supernova light curve, and other observable features of the supernova event can be affected by prior binary membership. Binary interactions may be the cause of much of the variability among type II supernova light curves. In particular, many of the peculiarities of SN 1987A and SN 1993J may well have resulted from the prior duplicity of the progenitors.
\end{abstract}

\section{Introduction}

A large fraction of all stars are members of binary systems. It is therefore reasonable to consider the possibility that the properties of many massive supernovae (i.e., supernovae whose progenitors had initial main-sequence masses greater than $\sim 8 \mathrm{M}_{\odot}$ ) are influenced by prior interactions of the progenitor with a binary companion star. This possibility was brought into focus in recent years by the nearby type II supernovae SN 1987A and SN 1993J, many of whose properties differed markedly from theoretical expectations. As a consequence, several studies have been undertaken to estimate the frequency of massive supernovae in binaries and to investigate the unique properties of the progenitors and supernova events that result from the evolution of a massive star in a close binary system (Podsiadlowski, Joss \& Hsu 1992, and references therein; Tutukov, Yungelson \& Iben 1992; 
Hsu et al. 1995). We here describe the main results of recent theoretical work on type II supernovae in binaries and discuss the application of this work to SN 1987A and SN 1993J. Work on the possible origin of at least some type Ib and Ic supernovae in binaries is reviewed elsewhere in these proceedings by Leibundgut (1995) and Nomoto (1995).

\section{Evolution of Type II Supernova Progenitors in Binaries}

The principal effects of evolution in a close binary system on the progenitor of a type II supernova can be broadly divided into three categories (Podsiadlowski, Joss \& Hsu 1992; hereafter PJH): (1) loss of a portion of the stellar envelope to the companion star, (2) accretion of matter from the companion star, or (3) merger of the two stars in a common-envelope phase. In addition, a star in a close but detached binary may lose a large fraction of its envelope in an enhanced stellar wind (Vanbeveren 1987; Tout \& Eggleton 1988).

On the basis of Monte Carlo calculations, PJH concluded that $\sim 20$ $35 \%$ of all massive stars experience binary interactions before undergoing a supernova explosion (which may be of type $\mathrm{Ib}$, Ic, or II). This is consistent with the findings of Tutukov et al. (1992), who concluded, by somewhat different means, that $\sim 25-45 \%$ of all supernovae (including those involving low-mass progenitors) originate in initially close binaries.

In the following paragraphs, we describe the salient features of each of the three modes of pre-supernova binary evolution listed above.

\subsection{MASS LOSS SCENARIOS}

If the supernova progenitor was originally the more massive of the binary components, it can lose mass to its companion via Roche-lobe overflow. This scenario has been considered in detail by Joss et al. (1988) and PJH. If the star first fills its Roche lobe while it is still on the main sequence, a contact system and eventual merger of the binary components is likely to result; the merged star should then have the properties of a rejuvenated main-sequence star. Of greater interest is the possibility that the primary first fills its Roche lobe during the course of its post-main-sequence evolution. In this case, the masses of the binary components will not be very different, and the resultant mass transfer can take place on a sufficiently long time scale that a common envelope does not form. In instances where the entire hydrogen-rich envelope is lost, the progenitor will become a helium star and will likely end its life as a type $\mathrm{Ib} / \mathrm{Ic}$ supernova (see $\mathrm{PJH}$; Nomoto 1995; and references in these works). If, however, the mass transfer process terminates when the progenitor still retains at least a few tenths of a solar mass of its envelope, the final pre-supernova radius and effective 
temperature will be nearly the same as those the star would have had in the absence of mass loss, and the resultant explosion will be of type II. Monte Carlo calculations ( $\mathrm{PJH}$ ) indicate that a few percent of all massive stars (perhaps up to $\sim 5 \%$ if systems with binary-enhanced winds are included) become type II supernovae of this class.

\subsection{ACCRETION SCENARIOS}

The original secondary in a close binary system with a Roche-lobe filling primary should accrete a substantial fraction of the mass lost by the primary. If the mass transfer commences before the original secondary has completed core hydrogen burning, the subsequent evolution of the secondary should mimic that of a more massive main-sequence star (Hellings 1983; PJH). Analogously to the case of mass-loss models, of greater interest is the situation where mass transfer commences only after the original secondary has left the main sequence (Podsiadlowski \& Joss 1989; De Loore \& Vanbeveren $1992 ; \mathrm{PJH})$. Due to the accreted mass, the original secondary becomes the more massive of the two stars, and its concomitantly accelerated evolution may cause it to reach the supernova stage prior to the original primary. If the original secondary is the first star to become a supernova, it will have a normal post-main-sequence companion at the time of the explosion; if, instead, the original primary reaches the supernova stage first, it should leave a neutron star or black-hole remnant that will remain gravitationally bound to the original secondary until the latter, too, becomes a supernova. In either case, however, the explosion of the original secondary will eject more than half of the residual systemic mass, generally causing the system to become unbound. Nevertheless, the companion object may become detectable after the supernova photosphere has receded sufficiently. Another diagnostic of supernova events of this class is the color of the immediate progenitor; if the original secondary accretes a sufficient amount of mass, it will end its life as a blue supergiant rather than a red supergiant, which is the generally expected precursor for a type II supernova that has evolved in isolation (see, e.g., Falk \& Arnett 1977; Woosley \& Weaver 1985).

\subsection{MERGER SCENARIOS}

If the initial masses of the two stars are sufficiently different, the time scale for mass transfer, once it commences, will be much less than the Kelvin time of the secondary. As a result, the mass transfer will be unstable, and the system will develop a common envelope (see, e.g., Paczyński 1976; Kippenhahn \& Meyer-Hofmeister 1977; Podsiadlowski, Joss \& Rappaport $1990 ; \mathrm{PJH})$; the primary should lose its entire hydrogen-rich envelope to the common envelope. Thereafter, dynamical friction between the secondary 
and the common envelope will cause the secondary to spiral in toward the system center-of-mass. It is uncertain whether or when the common envelope will subsequently be ejected (see Hsu et al. 1995 for a discussion). If the envelope is ejected before either the secondary is dissolved or the binary components merge to form a single star, and if the core mass of the primary is greater than $\sim 1.4 \mathrm{M}_{\odot}$ at the time of the ejection, a type $\mathrm{Ib} / \mathrm{Ic}$ event may result; however, if the explosion strips off a significant amount of the hydrogen-rich envelope of the secondary, the supernova may have the appearance of a type II event. In cases where mass transfer commences when the primary is still on the first red giant branch (case B transfer) and the common envelope is not subsequently ejected, the spiral-in time scale should be much shorter than the remaining evolutionary time for the primary; the binary components should therefore merge before a supernova event occurs. If, instead, mass transfer does not commence until the primary has reached the asymptotic giant branch (case $\mathrm{C}$ transfer) and the common envelope is not ejected, it is uncertain whether merger will occur before the the primary becomes a supernova. When the binary components merge before the occurrence of the supernova event, the net effect is very similar to that of the accretion scenario described in Section 2.2, and the merged star may well end its life as a blue supergiant. If the merger is not yet complete by the time of the supernova event, the immediate progenitor (i.e., the common envelope itself) may have the appearance of either a red or a blue supergiant, depending on the values of various parameters for the initial binary system and the details of the common-envelope evolution. Monte Carlo calculations (PJH) indicate that $\sim 3-6 \%$ of all massive stars end their lives as blue supergiants, due to accretion from or merger with a binary companion, before exploding as type II supernovae.

\section{Hydrodynamics of Massive Supernovae in Binaries}

We have recently completed a series of hydrodynamic calculations to explore the consequences of mass-loss and accretion/merger scenarios for the observational properties of the resultant type II supernova events (Hsu et al. 1995). We here briefly summarize the results of these calculations.

To investigate the effects of mass loss from a type II supernova progenitor, either through mass transfer to a close-binary stellar companion (see Section 2.1) or via a strong intrinsic stellar wind, we followed the explosion of a star with an initial main-sequence mass, $M_{\mathrm{ms}}$, of $12 \mathrm{M}_{\odot}$. In the absence of mass loss, such a star would have a hydrogen-rich envelope of mass $M_{\text {env }} \simeq 8.7 \mathrm{M}_{\odot}$; we considered cases with residual envelope masses of $0.4,1.9$, and $4.9 \mathrm{M}_{\odot}$, as well as a case with no mass loss.

The visual light curves of our four mass-loss models are shown in the 


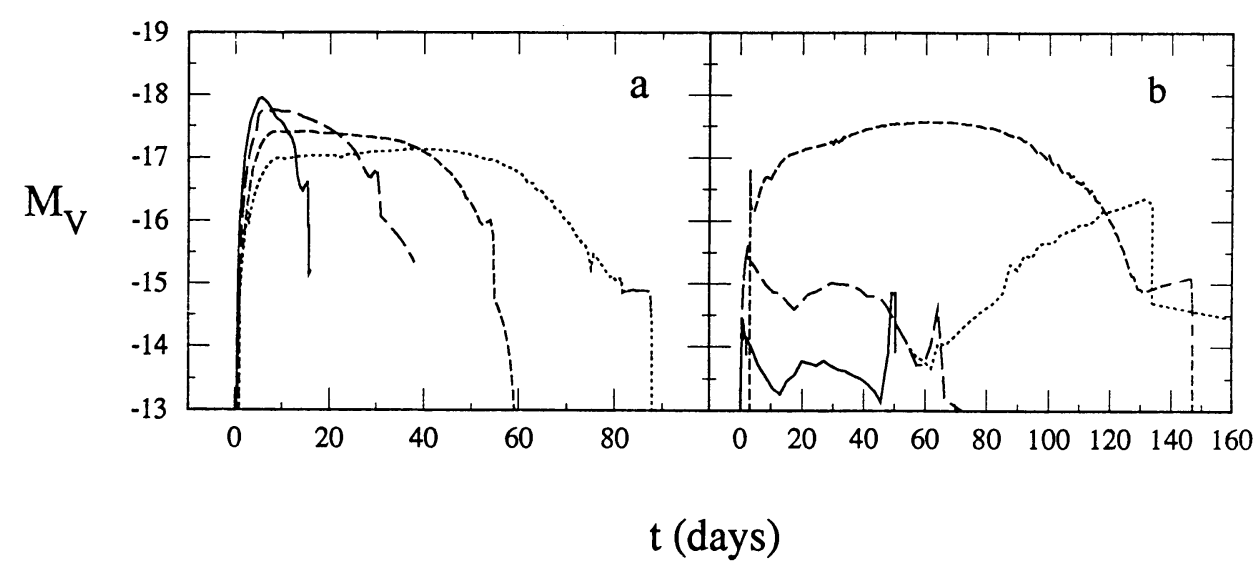

Figure 1. (a) Light curves (absolute visual magnitude, $M_{\mathrm{V}}$, as a function of elapsed time, $t$, since core collapse) for four supernova models whose progenitors had initial main-sequence masses, $M_{\mathrm{ms}}$, of $12 \mathrm{M}_{\odot}$ but had lost their hydrogen-rich envelopes to varying degrees during the course of their post-main-sequence evolution. All four hydrodynamic calculations assumed an explosion energy, $E$, of 1 foe and no energy input from the decay of radioactive material. Solid curve, $M_{\mathrm{env}}=0.4 \mathrm{M}_{\odot}$. Long-dashed curve, $M_{\mathrm{env}}=1.9 \mathrm{M}_{\odot}$. Short-dashed curve, $M_{\mathrm{env}}=4.9 \mathrm{M}_{\odot}$. Dotted curve, $M_{\mathrm{env}}=8.7 \mathrm{M}_{\odot}$ (corresponding to no mass loss). (b) Same as (a), but for three models whose progenitors underwent accretion from, or merger with, a binary stellar companion during the course of their post-main-sequence evolution; the final pre-supernova mass was $20 \mathrm{M}_{\odot}$ in all cases. All calculations again assumed $E=1$ foe and no energy input from radioactive decay, except where otherwise noted. Solid curve, $M_{\mathrm{ms}}=15 \mathrm{M}_{\odot}$. Long-dashed curve, $M_{\mathrm{ms}}=17 \mathrm{M}_{\odot}$. Dotted curve, $M_{\mathrm{ms}}=17 \mathrm{M}_{\odot}$, with additional energy from the radioactive decay of $0.071 \mathrm{M}_{\odot}$ of $\mathrm{Ni}^{56}$ and its decay product, $\mathrm{Co}^{56}$, deposited in the innermost layers of the ejecta; this light curve comes closest to matching the general properties of the light curve of SN 1987A, although it does not fit the observed light curve in detail (see Hsu et al. 1995 for a discussion). Short-dashed curve, $M_{\mathrm{ms}}=20 \mathrm{M}_{\odot}$, with no mass gained or lost by the progenitor during the course of its pre-supernova evolution, shown for comparison. (From Joss et al. 1994; adapted from Hsu et al. 1995.)

left-hand panel of Fig. 1. The most dramatic effects of a reduced envelope mass are (1) a much more rapid rate of decline of the light curve, (2) a higher peak luminosity (by as much as a magnitude in the $\mathrm{V}$ band), and (3) peak photospheric velocities that are higher by as much as a factor of $\sim 2\left(\sim 210^{4} \mathrm{~km} \mathrm{~s}^{-1}\right.$ for the model with the smallest residual envelope, compared to $\sim 110^{4} \mathrm{~km} \mathrm{~s}^{-1}$ for the model with no mass loss).

In order to explore the effects of an increase in the envelope mass of the progenitor via accretion from or merger with a binary companion during the course of its post-main-sequence evolution, we calculated the explosion of three stars, each with a final pre-supernova mass of $20 \mathrm{M}_{\odot}$. The first star had an initial main-sequence mass of $20 \mathrm{M}_{\odot}$ and underwent no mass loss or gain during the course of its evolution; the other two stars had $M_{\mathrm{ms}}=$ $17 \mathrm{M}_{\odot}$ and $15 \mathrm{M}_{\odot}$ and suffered a net gain of 3 and $5 \mathrm{M}_{\odot}$, respectively, 
during their post-main-sequence evolution. Both models that gained mass were blue supergiants, rather than red ones, at the time of the supernova event (see Sections 2.2 and 2.3, Podsiadlowski \& Joss 1989, and PJH).

The visual light curves for these three models are shown in the righthand panel of Fig. 1. The principal effects of the addition of mass are (1) a peak luminosity that is fainter by as much as 3.5 magnitudes in the $\mathrm{V}$ band (if we exclude the initial flash near $t=0$, which is not modeled very accurately in our calculations), (2) a more rapid decline of the light curve, and (3) a reduction of the peak photospheric velocity by as much as a factor of $\sim 2.5$, from $\sim 1.610^{4} \mathrm{~km} \mathrm{~s}^{-1}$ for the constant-mass model to only $\sim 610^{3} \mathrm{~km} \mathrm{~s}^{-1}$ for the model that has gained $5 \mathrm{M}_{\odot}$ during the course of its evolution.

\section{Application to Recent Supernovae}

\subsection{SN $1987 \mathrm{~A}$}

A number of authors (Fabian \& Rees 1988; Joss et al. 1988; Barkat \& Wheeler 1989; Hillebrandt \& Meyer 1989; Podsiadlowski \& Joss 1989; Podsiadlowski, Joss \& Rappaport 1990; De Loore \& Vanbeveren 1992; PJH; Rathnasree 1993) have explored the possibility that $\mathrm{Sk}-69^{\circ} 202$, the progenitor of SN 1987A, had been a member of a binary system prior to the supernova event. Among the various binary scenarios that have been proposed, the most promising appears to be one in which $\mathrm{Sk}-69^{\circ} 202$ underwent merger with a binary stellar companion in a common-envelope phase (Hillebrandt \& Meyer 1989; Podsiadlowski, Joss \& Rappaport 1990; $\mathrm{PJH}$ ). (The plausibility of accretion scenarios has been somewhat diminished by the lack of evidence for either a prior supernova event or a normal or neutron-star companion following the recession of the photosphere of SN 1987A.) The major lines of evidence in support of a merger scenario include (1) the blue color of $\mathrm{Sk}-69^{\circ} 202$, which was in contrast to most prior theoretical expectations (see Sections 2.2 and 2.3, Podsiadlowski \& Joss 1989 , and $\mathrm{PJH}),(2)$ chemical peculiarities in the progenitor and in the supernova ejecta, which may have resulted from the dredge-up of nuclearprocessed material during the merger process (see Hillebrandt \& Meyer 1989; PJH; and references in these works), (3) the low peak luminosity of SN 1987A and the exceptionally strong effect of energy input from radioactive decay upon its light curve, which is in accord with the results of hydrodynamic calculations by $\mathrm{Hsu}$ et al. (1995) for accretion/merger models of type II supernovae (see Section 3 and Fig. 1b), and (4) the approximate axial symmetry of the circumstellar material, which consists of a central ring (Crotts \& Heathcote 1991), two outer rings that are displaced approximately symmetrically on either side of the central ring and 
are approximately concentric with it (Lemonick 1994), and an outer, diffuse nebulosity known as "Napoleon's Hat" (Wampler et al. 1990). In regard to this last point, Cumming \& Podsiadlowski (1994) have recently presented a model for the triple-ring structure based on the hypothesis that $\mathrm{Sk}-69^{\circ} 202$ underwent merger with a binary companion in the not-too-distant past; this model can successfully explain not only the physical appearance of the rings but their measured velocity structure as well.

\section{2. $\mathrm{SN} 1993 \mathrm{~J}$}

Supernova 1993J, which exploded in the nearby spiral galaxy M81, was the brightest supernova since SN $1987 \mathrm{~A}$ and, like the latter, it was a type II event whose properties were peculiar in some key respects. A candidate progenitor, whose position was consistent with that of the supernova, displayed a blended spectrum that can be best fit by a late-B to early-A supergiant (or an OB association) and a $\mathrm{G}$ to early-K supergiant (Aldering, Humphreys \& Richmond 1994; see also Podsiadlowski et al. 1993); as in the case of SN 1987A, these spectral types are inconsistent with most theoretical expectations concerning the evolutionary state of the progenitor of a type II supernova that has evolved in isolation. (The actual progenitor may, however, have been a fainter star in the same field, especially if it had been the mass-losing component of an interacting binary; see Podsiadlowski et al. 1993.) Moreover, the initial peak of the supernova light curve was very sharp (qualitatively similar to the solid curve in Fig. 1a). This latter feature, in particular, led almost immediately to the suggestion by several authors (Nomoto et al. 1993; Podsiadlowski et al. 1993, 1994; Ray, Singh \& Sutaria 1993; Woosley et al. 1994) that the progenitor had lost most of its hydrogen-rich envelope by transfer to a close binary companion, in the manner discussed in Section 2.1 (see Fig. 2). This suggestion was confirmed by the subsequent evolution of the spectrum of the supernova from type II to type I (Filippenko, Matheson \& Ho 1993), indicating that the hydrogen-rich envelope was of anomalously low mass at the time of the explosion. (The alternative hypothesis that the progenitor had lost most of its hydrogen-rich envelope in a strong intrinsic stellar wind requires fine tuning, in order for the star to retain a small but non-vanishing amount of hydrogen at the time of the supernova event.)

We observe that there may be an interesting evolutionary link between SN 1993J and SN 1987A. If the companion of SN 1993J accreted several solar masses of material during the mass-transfer process, at a time when it had already evolved off the main sequence, it should end its life $\left(\sim 10^{5}-10^{6}\right.$ years hence) as a blue supergiant, and this second supernova event should resemble SN 1987A. 


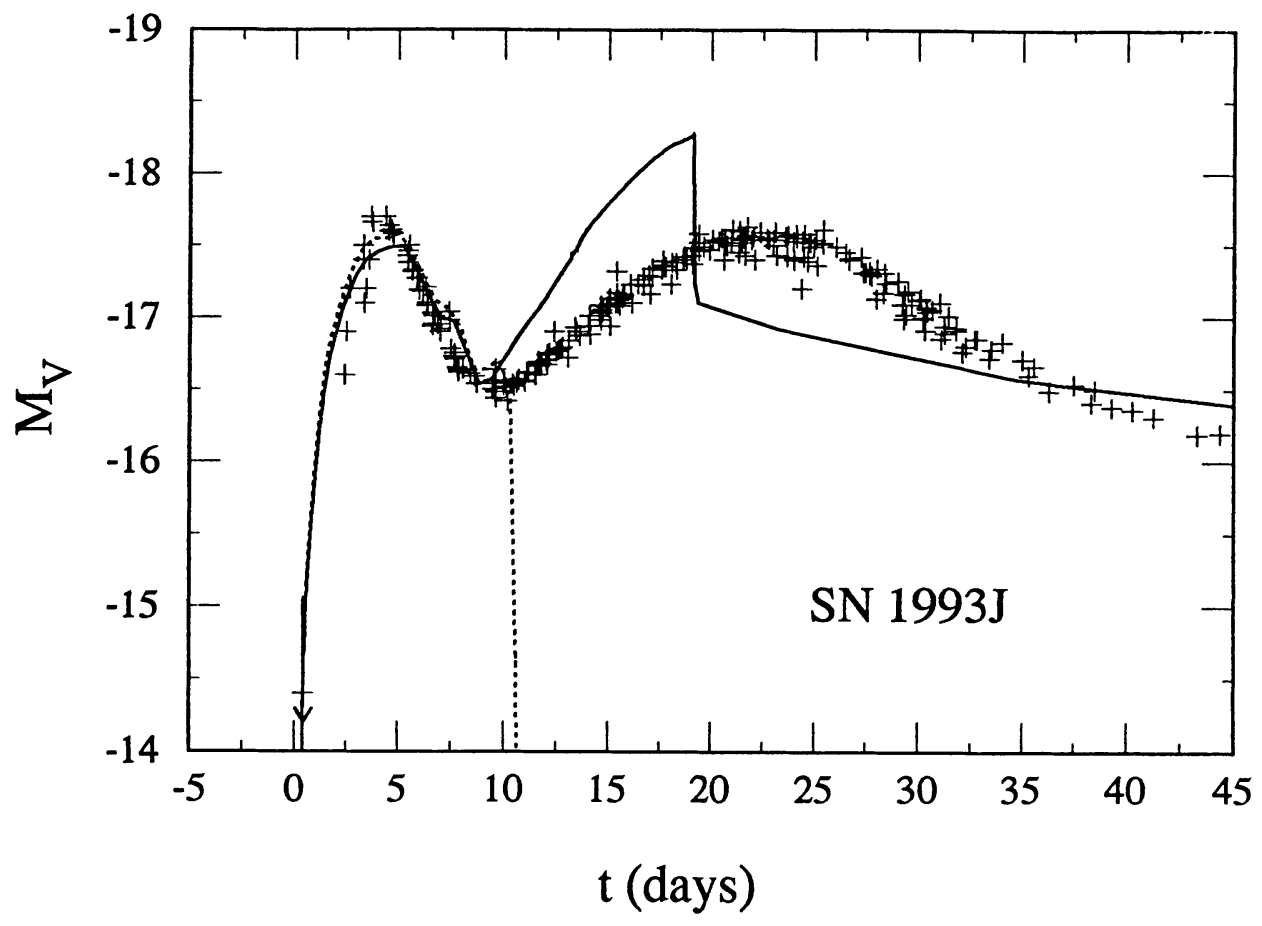

Figure 2. Comparison of theoretical visual light curves $\left(M_{\mathrm{V}}\right.$ as a function of $\left.t\right)$ with observations of SN 1993J. The time of core collapse $(t=0)$ corresponds to March 26.0 $\mathrm{UT}$, and the shock breaks out at $t=8.8 \mathrm{hr}$. The crosses represent observed $V$ magnitudes, as compiled by T. Kato and communicated by R.J. Cumming and A.V. Filippenko, which have been converted to absolute magnitudes by use of an assumed distance modulus to M81 of 27.6 mag and a visual extinction of $0.8 \mathrm{mag}$; the cross marked with an arrow near $t=0.5$ days is a lower limit on $M_{\mathrm{V}}$. For both theoretical curves, the progenitor was assumed to be a $\mathrm{K}$ supergiant which had an initial main-sequence mass of $15 \mathrm{M}_{\odot}$ but lost all but $0.2 \mathrm{M}_{\odot}$ of its hydrogen-rich envelope during the course of its post-main-sequence evolution. Dotted curve, model with $E=1$ foe and no input of energy from radioactive decay. Solid curve, model with $E=0.9$ foe plus energy from the radioactive decay of $0.15 \mathrm{M}_{\odot}$ of $\mathrm{Ni}^{56} / \mathrm{Co}^{56}$ deposited in the innermost layers of the ejecta. Several effects that were not taken into account in the theoretical calculations, including Rayleigh-Taylor instabilities (which may have produced clumping of the ejecta and enhanced opacities within the inner ejecta due to the admixture of a small amount of hydrogen), would tend to smooth the light curve for $t>10$ days and thereby produce a better fit between the latter theoretical light curve and the observed light curve. (From Hsu et al. 1995; see also Podsiadlowski et al. 1993.)

It is remarkable that both SN 1987A and SN 1993J, the two nearest known supernovae of the past century, have both displayed substantial evidence for origin in massive close-binary systems. Of course, evidence of prior duplicity becomes easier to obtain with increasing proximity of the supernova event. It is distinctly possible that, for supernovae of all types, prior duplicity will turn out to be the rule rather than the exception. 
Acknowledgements. It is a pleasure to acknowledge my collaborators J. Hsu, Ph. Podsiadlowski, S. Rappaport, and R. Ross, whose efforts were essential to the completion of all aspects of the work described in this article. This work was supported in part by the U.S. National Aeronautics and Space Administration under grant NAGW-1545.

\section{References}

Aldering, G., Humphreys, R.M. \& Richmond, M. 1994, AJ 107, 662

Barkat, Z. \& Wheeler, J.C. 1989, ApJ 342, 940

Crotts, A.P.S. \& Heathcote, S.R. 1991, Nat 350, 683

Cumming, R.J. \& Podsiadlowski, Ph. 1994, (preprint)

De Loore, C. \& Vanbeveren, D. 1992, A\&A 260, 273

Fabian, A.C. \& Rees, M.J. 1988, Nat 335, 50

Filippenko, A.V., Matheson, T. \& Ho, L.C. 1993, ApJ 415, L103

Falk, S.W. \& Arnett, W.D. 1977, ApJS 33, 515

Hellings, P. 1983, Ap\&SS 96, 37

Hillebrandt, W. \& Meyer, F. 1989, A\&A 219, L3

Hsu, J.J.L., Joss, P.C., Ross, R.R. \& Podsiadlowski, Ph. 1995, ApJ (in press)

Joss, P.C., Hsu, J.J.L., Podsiadlowski, Ph. \& Ross, R.R. 1994, in Proc. 34th Herstmonceux Conf., Circumstellar Media in the Late Stages of Stellar Evolution,

R. Clegg et al. (Eds.), Cambridge Univ. Press (Cambridge, England), (in press)

Joss, P.C., Podsiadlowski, Ph., Hsu, J.J.L. \& Rappaport, S. 1988, Nat 331, 237

Kippenhahn, R. \& Meyer-Hofmeister, E. 1977, A\&A 54, 539

Leibundgut, B. 1995, these Proceedings

Lemonick, M.D. 1994, Time, Vol. 143, No. 22, 51

Nomoto, K. 1995, these Proceedings

Nomoto, K. et al. 1993, Nat 364, 507

Paczyński, B. 1976, in IAU Symp. No. 73, Structure and Evolution of Close Binary Systems, P.P. Eggleton et al. (Eds.), Reidel (Dordrecht), p. 75

Podsiadlowski, Ph. \& Joss, P.C. 1989, Nat 338, 401

Podsiadlowski, Ph., Hsu, J.J.L., Joss, P.C. \& Ross, R.R. 1993, Nat 364, 509

Podsiadlowski, Ph., Hsu, J.J.L., Joss, P.C. \& Ross, R.R. 1994, in Proc. 34th Herstmonceux Conf., Circumstellar Media in the Late Stages of Stellar Evolution, R. Clegg et al. (Eds.), Cambridge Univ. Press (Cambridge, England), (in press)

Podsiadlowski, Ph., Joss, P.C. \& Hsu, J.J.L. 1992, ApJ 391, 246 (PJH)

Podsiadlowski, Ph., Joss, P.C. \& Rappaport, S. 1990, A\&A 227, L9

Rathnasree, N. 1993, ApJ 411, 848

Ray, A., Singh, K.P. \& Sutaria, F.K. 1993, J. Astrophys. Astr. 14, 53

Tout, C.A. \& Eggleton, P.P. 1988, ApJ 334, 357

Tutukov, A.V., Yungelson, L.R. \& Iben, I. 1992, ApJ 386, 197

Vanbeveren, D. 1987, A\&A 182, 207

Wampler, E.J. et al. 1990, ApJ 362, L13

Woosley, S.E. \& Weaver, T.A. 1985, in Nucleosynthesis and Its Implications On Nuclear and Particle Physics, Proc. 5th Moriand Astrophys. Conf., J. Audouze \& T. van Thuan (Eds.), Reidel (Dordrecht), p. 145

Woosley, S.E., Eastman, R.G., Weaver, T.A. \& Pinto, P.A. 1994, ApJ 429, 300 